\title{
Resonant Raman scattering in cubic and hexagonal boron nitride
}

\author{
S. Reich and A. C. Ferrari \\ University of Cambridge, Department of Engineering, Trumpington Street, Cambridge CB2 1PZ, United Kingdom \\ R. Arenal and A. Loiseau \\ LEM, ONERA-CNRS, BP72, 92322 Châtillon Cedex, France \\ I. Bello \\ Department of Physics and Materials Science, City University of Hong Kong, Hong Kong, China \\ J. Robertson \\ University of Cambridge, Department of Engineering, Trumpington Street, Cambridge CB2 1PZ, United Kingdom
}

(Received 25 August 2004; published 4 May 2005)

\begin{abstract}
We measured first- and second-order Raman scattering in cubic and hexagonal boron nitride using excitation energies in the visible and in the UV. The nonresonant first-order Raman susceptibilities for cubic and hexagonal $\mathrm{BN}$ are 1 and $10 \AA^{2}$, respectively. Raman scattering is thus very powerful in detecting the hexagonal phase in mixed thin boron nitride films. In cubic BN the constant Raman sucseptibility in the visible and the $\mathrm{UV}$ is due to its indirect band gap. For hexagonal BN a Raman enhancement is found at $5.4 \mathrm{eV}$. It is well explained by the energy dependence of the dielectric function of hexagonal BN. The second-order spectrum of cubic boron nitride is in excellent agreement with first-principles calculations of the phonon density of states. In hexagonal $\mathrm{BN}$ the overbending of the $\mathrm{LO}$ phonon is $\approx 100 \mathrm{~cm}^{-1}$, five times larger than in graphite.
\end{abstract}

DOI: 10.1103/PhysRevB.71.205201

PACS number(s): 78.30.Fs, 63.20.Dj

\section{INTRODUCTION}

Boron nitride exists in different polytypes, the $s p^{2}$ bonded hexagonal and rhombohedral structures and the $s p^{3}$ cubic and wurzite phases. ${ }^{1,2}$ Hexagonal and cubic BN are analogous to graphite and diamond, respectively. All phases are wide band-gap semiconductors $(>5 \mathrm{eV})$. In particular, the electronic structure of hexagonal $\mathrm{BN}$ and graphite are very different despite their structural similarities. Graphite is electrically conductive, whereas hexagonal $\mathrm{BN}$ is insulating with a band gap of $5 \mathrm{eV} .^{3}$ This is caused by the ionic character of this material. Thin boron nitride films often contain amorphous and disordered turbostratic boron nitride structures.

Cubic boron nitride with its zinc blende lattice is isostructural and isoelectronic to diamond. ${ }^{4}$ Its properties are similar to diamond and in some aspects it surmounts diamond. Unlike diamond it is chemically inert to ferrous materials at high temperature and chemically and thermally far more stable. ${ }^{5}$ Cubic boron nitride has the second-highest hardness and thermal conductivity of any material as well as a low friction coefficient. All these properties make cubic BN the best material for machining ferrous materials and for coatings in tribological applications. On the other hand, the electronic and thermal properties of cubic BN suggest to use it in electronic applications. Because of its large band gap, cubic $\mathrm{BN}$ could be used for high-power-high-frequency electronic devices. These devices can potentially operate in harsh, hightemperature, and radiative environments. Another advantage of cubic $\mathrm{BN}$ is the ease of $p$ - and $n$-type doping. ${ }^{6,7}$ This is still problematic in diamond.

Cubic boron nitride is synthesized in powders with crystallites ranging from submicron size up to a size of $3 \mathrm{~mm} .^{8,9}$ The crystallites are industrially used as abrasives. They are also sintered to tools of desired shapes using metal binders. Thin films of cubic BN are grown by vapor-deposition methods. The films, however, show drawbacks, especially, a low crystallinity and high defect density. Other problems are the presence of interfacial soft hexagonal, disordered turbostratic, and amorphous BN layers, and a low phase purity. Moreover, high internal stresses as normally found in cubic BN films limit the ultimate film thickness. All this prevented the application of thin boron nitride films. Recently great success was made in the quality of cubic BN films by growing cubic BN epitaxially on high-quality CVD diamond films and polycrystalline diamond. ${ }^{10-12}$ Zhang et al. ${ }^{10}$ reported 30 -nm-thick films of high crystallinity and purity as evidenced by a variety of structural measurements.

Hexagonal $\mathrm{BN}$ received much attention recently after the discovery of boron nitride nanotubes. ${ }^{13-16}$ These tubes consist of one or several layers of hexagonal BN wrapped up into a hollow cylinder. The relation between $\mathrm{BN}$ nanotubes and hexagonal $\mathrm{BN}$ is thus the same as the relation between carbon nanotubes and a single sheet of graphite. ${ }^{17}$ The nanotube properties can be derived by studying the wrapping of a single layer of the hexagonal bulk material. Boron nitride nanotubes show some important differences to the widely studied carbon nanotubes. For example, the electrical properties of carbon nanotubes change dramatically from tube to tube. Depending on their diameter and chiral angle carbon nanotubes are semiconductors or metals. ${ }^{17,18}$ Moreover, tubetube interaction strongly alters their electronic properties. ${ }^{19}$ Boron nitride nanotubes, in contrast, are always semiconducting and their band gaps are rather insensitive to the chiral angle of the tube and tube-tube interaction. ${ }^{20-22}$ For diameters above $\approx 9.5 \AA \mathrm{BN}$ nanotubes were predicted to have a constant band gap around $5.5 \mathrm{eV} .^{20-22}$ Boron nitride nano- 
tubes have outstanding elastical properties and are chemically intert. ${ }^{23,24}$ They can thus be envisioned as a protective cage in the nanoworld.

The phonon frequencies of boron nitride nanotubes were recently calculated by Sánchez-Portal and Hernández ${ }^{25}$ and Wirtz et al. ${ }^{26}$ Both groups found good agreement between a zone-folding approximation and a full calculation for several BN nanotubes; similar conclusions were drawn from a valence shell model. ${ }^{27}$ Within zone folding the $\Gamma$ point vibrations of a nanotube originate from non- $\Gamma$-point vibrations of hexagonal BN. A better knowledge of the phonon dispersion of hexagonal boron nitride is thus highly desirable to model and understand the properties of BN nanotubes.

Another reason for the high interest in boron nitride allotropes are the variety of phase transitions in this compound. Several theoretical studies have been devoted to the phonon dispersion, the Grüneisen parameters, the free energy from the phonon density of states, and the phase diagram. ${ }^{28,29}$ These theoretical works on the phonon dispersion of cubic and hexagonal $\mathrm{BN}$, however, have never been challenged experimentally. Only the $\Gamma$ point phonon frequencies are known from Raman and infrared spectroscopy. ${ }^{30-33}$

The development and production of boron nitride for possible applications need reliable and quick analytical characterization. In particular, questions such as the crystalline quality and the presence of impurity phases have to be addressed. Raman and infrared spectroscopy are quick and nondestructive tools to assess the structural properties of materials. Infrared spectroscopy is the most widely used for BN, since it is very sensitive to the polar boron-nitride bonds and the cubic and hexagonal forms have distinct infrared features. ${ }^{28-33}$ However, infrared spectroscopy has limitations. The substrate has to be either transparent or not highly reflective. Whereas the silicon substrates used nowadays meet these two requirements, it is difficult to investigate films on more interesting substrates such as, e.g., iron steel for highduty tools.

Raman spectroscopy is rarely used for boron nitride because of its inferior sensitivity, especially, in the case of thin films. This is opposite to diamondlike carbons, where Raman scattering is a standard characterization tool. ${ }^{34,35}$ To successfully apply this technique in boron nitrides its low sensitivity has to be overcome. Raman scattering also holds the potential of measuring the ratio between cubic and hexagonal BN in a sample. The scattering intensity is proportional to the volume fraction of the two phases. To apply this idea we need to know the relative Raman efficiency of the cubic and hexagonal form. ${ }^{36}$

Second-order Raman scattering probes phonons away from the $\Gamma$ point of the Brillouin zone. It can be used to measure the phonon dispersion of a material via its phonon density of states. Second-order Raman scattering is thus a powerful test for the calculated phonon dispersion of hexagonal and cubic BN.

In this paper we study Raman scattering on cubic and hexagonal boron nitride using UV $(5.08$ and $5.41 \mathrm{eV})$ and visible $(2.41 \mathrm{eV})$ excitation energies. We determine the absolute Raman cross section from the LO-TO ratio in cubic $\mathrm{BN}$ and by comparing the Raman intensity of $\mathrm{BN}$ and diamond. For cubic BN the Raman susceptibility is constant up to $5.41 \mathrm{eV}$. We show that this is due to the indirect band gap that does not yield a resonant enhancement. In hexagonal BN we find an enhancement by a factor of 2-5 when comparing the Raman susceptibility in the visible and the UV. The measured Raman cross section is in excellent agreement with the derivative of the dielectric function. The comparatively weak resonance in hexagonal $\mathrm{BN}$ at the fundamental gap is a consequence of the large impurity-related absorption in the visible. The Raman efficiency of hexagonal BN is two to three orders of magnitude larger than in the cubic phase. Combined with the $\omega^{4}$ enhancement of the scattering efficiency, this makes Raman scattering very promising for detecting the hexagonal phase in nominally cubic boron nitride films. In addition to the Raman-active first-order lines we measured the second-order Raman spectrum. For cubic boron nitride we find excellent agreement between the second-order spectrum and first-principles calculations of the phonon density of states. In hexagonal boron nitride the comparison between experiment and theory is worse. While the high-energy optical phonons are excellently described by existing $a b$ initio calculations, the ionic interaction appears to be strongly underestimated for lower energies. In particular, the rigid vibration of the boron nitride planes has an energy of $310 \mathrm{~cm}^{-1}$, twice as much as expected. Large deviations are also found for the ZA and LA phonons at the boundary of the Brillouin zone. We also discuss the origin of the mode at $920 \mathrm{~cm}^{-1}$ in boron nitride, which we assign to an overtone of a zoneboundary phonon in the hexagonal $\mathrm{BN}$ phase.

This paper is organized as follows. In Sec. II we describe our experimental setup and the samples used for the present study. The first-order Raman spectra are presented in Sec. III. In Sec. IV we determine the absolute Raman cross section from the LO-TO ratio in cubic BN (Sec. IV A) and by comparing the BN Raman spectra to diamond (Sec. IV B). Resonant Raman scattering in boron nitride is discussed in Sec. IV C. In Sec. V we present the second-order Raman spectra of cubic (Sec. V A) and hexagonal (Sec. V B) BN and compare them to ab initio calculations of the phonon density of states. Section VI summarizes our work.

\section{EXPERIMENTS}

UV Raman experiments were excited with the 244 $(5.08 \mathrm{eV})$ and $229 \mathrm{~nm}(5.41 \mathrm{eV})$ lines of an intracavity frequency doubled $\mathrm{Ar}^{+}$laser. The light was focused with a microscope onto a spot size of $\approx 1 \mu \mathrm{m}$ in diameter. The scattered light was collected in backscattering geometry and dispersed by a Renishaw Raman System 1000 spectrometer modified for the use in the UV with fused silica optics. The spectra were detected with a UV-enhanced charged couple device (CCD) camera. The spectral resolution was $8 \mathrm{~cm}^{-1}$. In this energy range the spectral response of the spectrometer is strongly polarized. The by far dominant contribution to the Raman spectra comes from parallel polarization of the incoming and scattered light. For visible excitation we used the $514.5 \mathrm{~nm}(2.41 \mathrm{eV})$ line of an $\mathrm{Ar}^{+}$laser. The focal spot of the microscope objective was $1 \mu \mathrm{m}$ in diameter. The spectra were detected with a Renishaw single-grating spectrometer equipped with a CCD. 


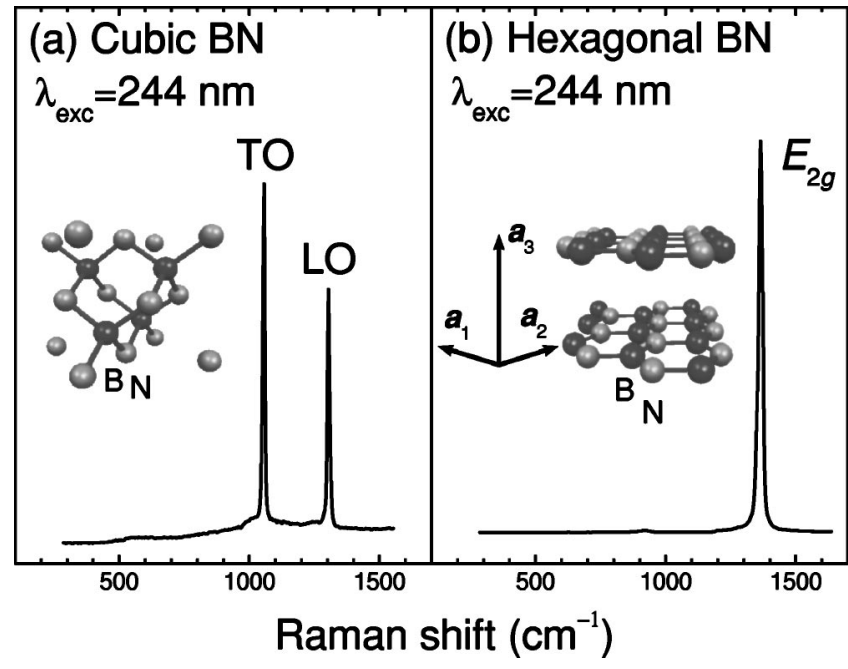

FIG. 1. First-order Raman spectra of (a) cubic and (b) hexagonal boron nitride excited with $\lambda_{\mathrm{exc}}=244 \mathrm{~nm}$. The inset shows the crystal structure of the two $\mathrm{BN}$ structures. Note the $A A^{\prime} A A^{\prime}$ stacking in the hexagonal type.

The sample of hexagonal boron nitride was obtained commercially (Lesker). It had a purity of $99.9 \%$. The cubic boron-nitride sample was produced by nucleation under high pressure (4.2 GPa) and temperature (1800-1900 K) using an $\mathrm{MgBN}$ catalyst system. Both samples were powders of small crystals. We selected single crystals under the optical microscope. For the hexagonal BN the direction of the incoming and scattered light was parallel to the $c\left(\boldsymbol{a}_{3}\right)$ axis. The high quality of the crystals was confirmed by the absence of the infrared-active phonons in our measurements. Cubic boron nitride crystals showed under the microscope a triangular shape, which is typical for the (111) surface of the zinc blende structure. To confirm that the incoming light was normal to the (111) surface we performed polarized Raman experiment in the visible, i.e., very far from any resonance. Both the LO and the TO phonon were observed in parallel polarization. In crossed polarization the TO peak was reduced by a factor of $\approx 2 / 3$ and the LO by $\approx 1 / 15$. This is in excellent agreement with the selection rules of the (111) surface in the zinc blende structure. ${ }^{37}$ The rather strong leakage of the LO mode in crossed polarization is typical for micro Raman scattering. It is due to the large collection angle of the microscope objective.

For the second-order Raman spectra we calculated the reduced Raman cross section by dividing the experimentally obtained intensities by $\hbar \omega_{s}^{3}\left[N\left(\omega_{\mathrm{ph}}\right)+1\right]^{2} / \hbar \omega_{\mathrm{ph}}$, where $\hbar \omega_{s}$ is the energy of the scattered photons, $\hbar \omega_{\mathrm{ph}}$ is the phonon energy, and $N\left(\omega_{\mathrm{ph}}\right)$ is the Bose-Einstein occupation number. ${ }^{37}$ These corrections are also taken into account for the firstorder lines. In contrast to the second-order spectra, however, we include them explicitly in the analysis of Sec. IV.

\section{FIRST-ORDER RAMAN SPECTRA}

Figure 1 shows the first-order Raman spectra of (a) cubic and (b) hexagonal boron nitride excited with $244 \mathrm{~nm}$. The insets display the crystal structures of the two BN polytypes. A detailed comparison of the Raman cross section obtained with UV and visible excitation will be presented in Sec. IV.

Cubic boron nitride belongs to the zinc blende structure. It has one optical phonon at $\Gamma$, which is Raman active $\left(\Gamma_{15}\right.$ or $T_{2}$ ). This triply degenerate mode splits into a transverse and longitudinal phonon because of the ionic character of $\mathrm{BN}$. The TO phonon is at $1055 \mathrm{~cm}^{-1}$ and the LO at $1304 \mathrm{~cm}^{-1}$ in Fig. 1(a) in excellent agreement with previous measurements. ${ }^{32,38,39}$ In Sec. IV we use the intensity ratio between the LO and TO scattering intensity to determine the absolute Raman cross section of cubic BN in the visible and the UV.

Hexagonal boron nitride is structually related to graphite. It consists of hexagonal planes of $\mathrm{B}$ and $\mathrm{N}$ atoms. In contrast to graphite, the stacking sequence is $A A^{\prime}$; the $\mathrm{B}$ atoms in one plane are on top of the $\mathrm{N}$ atoms in the other plane and vice versa, see inset of Fig. 1(b). The Raman-active high-energy phonon is at $1364 \mathrm{~cm}^{-1} \cdot 30,31$ The phonon eigenvector of this mode is a doubly degenerate in-plane optical mode with $\Gamma_{5}^{+}$ or $E_{2 g}$ symmetry. The boron and the nitrogen atoms in each plane move in opposite directions. The atomic displacements in the two planes are combined symmetrically. Interestingly, this mode does not have an LO-TO splitting, since the contributions from the two planes cancel each other. The antisymmetric combination of the high-energy in-plane mode is infrared active $\left(\Gamma_{6}^{-}\right.$or $\left.E_{1 u}\right)$ and has a large LO-TO splitting of $\approx 240 \mathrm{~cm}^{-1}$. The infrared-active TO mode was observed at $1367 \mathrm{~cm}^{-1}$, the LO at $1612 \mathrm{~cm}^{-1} \cdot 30$

Hexagonal $\mathrm{BN}$ has a second Raman-active vibration at very low frequencies $\left(55 \mathrm{~cm}^{-1}\right.$, Ref. 31$)$. This phonon, where the two BN planes slide against each other, was not detectable in our experiment due to the cutoff of the notch filter at $\approx 450 \mathrm{~cm}^{-1}$. The second low-energy vibration-a rigid movement of the planes against each other parallel to $\boldsymbol{a}_{3}$-is neither Raman nor infrared active $\left(\Gamma_{4}^{+}\right.$or $\left.B_{2 g}\right)$. It is predicted at $125 \mathrm{~cm}^{-1}$ from $a b$ initio calculations. Finally, in the intermediate frequency range around $800 \mathrm{~cm}^{-1}$ there are two outof-plane optical modes. One is the $\Gamma_{2}^{+}\left(A_{2 u}\right)$ infrared-active phonon, the other is the second silent vibration with $\Gamma_{4}^{+}$or $B_{2 g}$ symmetry, see also Sec. V B, in particular, the phonon dispersion of hexagonal $\mathrm{BN}$ in Fig. 5.

\section{ABSOLUTE RAMAN CROSS SECTION OF CUBIC AND HEXAGONAL BN}

There are several methods to determine the Raman cross section of a material. ${ }^{37}$ The most widely used is sample substitution, where the Raman signal of the sample under study is compared to the Raman signal of a reference material with a known efficiency. Zinc blende materials provide the alternative way to measure the Raman cross section from the ratio of the LO and the TO scattering intensity. ${ }^{37}$ We will use both methods, starting with a calculation of the Raman polarizability from the LO-TO ratio in cubic BN.

\section{A. LO-TO ratio in cubic boron nitride}

In Raman backscattering from the (111) surface of cubic $\mathrm{BN}$ both the TO and the LO phonon are allowed by selection 
rules. In parallel polarization and an excitation energy of $5.08 \mathrm{eV}(244 \mathrm{~nm})$ an intensity ratio $I_{\mathrm{LO}} / I_{\mathrm{TO}}=0.63$ is expected if the Raman cross section for LO and TO scattering are the same. As can be seen in Fig. 1(a) the experimental ratio is somewhat larger. The LO phonons are enhanced because of the electrical field accompanying a longitudinal vibration. Transverse optical phonons can only scatter through deformation-potential interaction. Longitudinal vibrations additionally interact with light through the electro-optical effect. ${ }^{37,40,41}$ Thus, the ratio between the LO and the TO scattering intensities measures the ratio between these two types of interactions. If the electro-optical coefficient of a material is known independently, the Raman susceptibility can be directly obtained from the LO-TO ratio.

Within this approach the absolute Raman susceptibility $a$ is given by 37,42

$$
a=\frac{V_{c}}{2} \frac{\partial \chi}{\partial u}=\frac{V_{c}}{2} \frac{\mu \omega_{\mathrm{TO}}^{2} \partial \chi / \partial E}{e^{*}} C,
$$

where $V_{c}$ is the volume of the unit cell, $\mu$ the reduced mass of the atoms in the cell, $\omega_{\mathrm{TO}}$ the TO phonon frequency, $e^{*}$ $=1.98 e$ the Born effective charge of cubic BN, $C$ the socalled Faust-Henry coefficient, and $\partial \chi / \partial E$ the electro-optical coefficient. The material parameters for cubic $\mathrm{BN}$ can be found in Table II in Sec. IV B. The electro-optical coefficient of cubic boron nitride $\partial \chi / \partial E=(1.3 \pm 0.5) \times 10^{-12} \mathrm{~m} / \mathrm{V}$ is only known from $a b$ initio calculations. ${ }^{43,44}$ For most III-V semiconductors and also for wurzite GaN and AlN the agreement between experiment and first-principles calculations of the electro-optical coefficient is very good; we therefore adopt the $a b$ initio value for the following analysis.

The Faust-Henry coefficient $C$ is obtained from the intensity ratio between the $\mathrm{TO}$ and $\mathrm{LO}$ phonons ${ }^{37,42}$

$$
C=-\left(\left|\frac{d_{\mathrm{LO}}}{d_{\mathrm{TO}}}\right|-1\right)^{-1} \frac{\omega_{\mathrm{LO}}^{2}-\omega_{\mathrm{TO}}^{2}}{\omega_{\mathrm{TO}}^{2}}
$$

where

$$
\left|\frac{d_{\mathrm{LO}}}{d_{\mathrm{TO}}}\right|^{2}=2 \frac{I_{\mathrm{LO}}}{I_{\mathrm{TO}}} \cdot \frac{\omega_{\mathrm{LO}}}{\omega_{\mathrm{TO}}} \cdot \frac{\left(\omega_{l}-\omega_{\mathrm{TO}}\right)^{3}}{\left(\omega_{l}-\omega_{\mathrm{LO}}\right)^{3}},
$$

for backscattering from the (111) surface and parallel polarization. $\omega_{l}$ is the laser frequency, $I_{\mathrm{LO}, \mathrm{TO}}$ are the integrated intensities of the LO and TO phonons, and $\omega_{\mathrm{LO}, \mathrm{TO}}$ are the phonon frequencies.

From the measured LO-TO intensity ratio at 2.41, 5.08, and $5.41 \mathrm{eV}$ we obtain an absolute Raman susceptibility $a$ $=(1.5 \pm 0.6) \AA^{2}$ for cubic boron nitride. The susceptibility is independent of the laser energy, see Table I, implying that no resonance or only a weak resonance occurs at the indirect band gap $E_{g} \approx 6.3 \mathrm{eV} .{ }^{45}$ Before discussing resonances in $\mathrm{BN}$ in more detail, we present our measurements of the Raman cross section in cubic and hexagonal $\mathrm{BN}$ by the method of sample substitution.

\section{B. Sample substitution with diamond}

At 2.41 and $5.08 \mathrm{eV}$ excitation energy we compared the Raman intensity of cubic and hexagonal boron nitride to the
TABLE I. Raman susceptibility $a$ and scattering efficiency $d S / d \Omega$ of cubic boron nitride from the intensity ratio $I_{\mathrm{LO}} / I_{\mathrm{TO}}$ of the LO and TO phonon. The error in the susceptibility and the efficiency includes the large uncertainty of the electro-optical coefficient. When considering only the statistical error of the measured intensities the uncertainty of $a$ is much smaller $\left(0.2 \AA^{2}\right.$ instead of $\left.0.5 \AA^{2}\right)$.

\begin{tabular}{cccc}
\hline \hline & & & $d S / d \Omega$ \\
$E_{\text {exc }}$ & $I_{\mathrm{LO}} / I_{\mathrm{TO}}$ & $a\left(\AA^{2}\right)$ & $\left(10^{-5} \mathrm{~m}^{-1} \mathrm{sr}^{-1}\right)$ \\
\hline 2.41 & $0.70 \pm 0.03$ & $1.5 \pm 0.5$ & $0.9 \pm 0.3$ \\
5.08 & $0.75 \pm 0.06$ & $1.4 \pm 0.6$ & $17 \pm 6$ \\
5.41 & $0.73 \pm 0.06$ & $1.5 \pm 0.6$ & $25 \pm 6$ \\
\hline \hline
\end{tabular}

scattering intensity of diamond. We used diamond as a reference because of its high Raman frequency $\left(1333 \mathrm{~cm}^{-1}\right)$. Normally, flourides $\left(\mathrm{CaF}_{2}\right.$ or $\left.\mathrm{BaF}_{2}\right)$ are taken as a reference in Raman scattering. ${ }^{37}$ Their low phonon frequencies $<400 \mathrm{~cm}^{-1}$, however, are cut by the notch filter in the UV measurements. However, Calleja et al. ${ }^{46}$ showed that the $E_{0}^{\prime}=7.4 \mathrm{eV}$ resonance in diamond vanishes by destructive interference with higher-lying optical transitions. Thus, the Raman suceptibility of diamond is to first approximation constant in the visible and the UV making it a good reference for Raman scattering.

We first briefly summarize the relation between the Raman susceptibility, the scattering efficiency and the intensity measured in a Raman experiment. ${ }^{37,47,48}$ For a given material and excitation energy the Raman scattering efficiency $d S / d \Omega$ can be calculated from the Raman susceptibility $a$ by

$$
\frac{d S}{d \Omega}=\frac{\omega_{l}\left(\omega_{l}-\omega_{\mathrm{ph}}\right)^{3}}{c^{4}} \frac{\hbar\left[N\left(\omega_{\mathrm{ph}}\right)+1\right]}{2 V_{c} \mu \omega_{\mathrm{ph}}} a^{2} .
$$

$V_{c}$ and $\mu$ are the volume and the reduced mass of the unit cell, $\omega_{l}$ the laser frequency, $\omega_{\mathrm{ph}}$ the phonon frequency, $N\left(\omega_{\mathrm{ph}}\right)$ the Bose-Einstein function, and $c$ the speed of light. We assumed a constant index of refractive at the energy of the incoming and scattered light for a given laser energy. ${ }^{48}$ If we approximate $\left(\omega_{l}-\omega_{\mathrm{ph}}\right) \approx \omega_{l}$ the scattering efficiency in Eq. (4) increases as $\omega_{l}^{4}$ for a constant Raman susceptibility, which is known as the $\omega^{4}$ dependence of the Raman cross section.

In a Raman experiment $d S / d \Omega$ is not directly accessible. What is measured are the number of scattered photons $R_{s}$ outside the crystal within a solid collection angle $\Delta \Omega$. The latter is fixed by the experimental setup. Additionally, the Raman intensity is referenced to the incoming laser power $P_{l}$. Correcting for the index of refraction, reflection losses upon entering and leaving the sample, and expressing $R_{s}$ as a function of measurable quantities one finds

$$
R_{s}=\frac{16}{\hbar \omega_{l}} P_{l} \Delta \Omega L \frac{d S}{d \Omega}
$$


TABLE II. Raman susceptibility of cubic and hexagonal BN as measured by sample substitution. The table also lists the material parameters necessary for the correction of the measured intensities. The Raman susceptibility of diamond was extrapolated to $5.08 \mathrm{eV}$ from the energy dependence reported by Calleja et al. (Ref. 46). The focal length of the optical miscroscope was assumed to be $1 \mu \mathrm{m}$. The scattering efficiency $d S / d \Omega$ is given in $10^{-5} \mathrm{~m}^{-1} \mathrm{Sr}^{-1}$.

\begin{tabular}{|c|c|c|c|c|}
\hline & & Cubic BN & Hexagonal BN & Diamond \\
\hline$\omega_{\mathrm{ph}}\left(\mathrm{cm}^{-1}\right)$ & & 1055 & 1364 & 1333 \\
\hline$V_{c}\left(\AA^{3}\right)$ & & 11.82 & 36.17 & 11.35 \\
\hline$\mu$ (a.u.) & & 6.10 & 3.05 & 6.00 \\
\hline \multicolumn{5}{|c|}{$514.5 \mathrm{~nm}, 2.41 \mathrm{eV}$} \\
\hline$n$ & (Ref. 49 and 50) & 2.1 & 1.8 & 2.4 \\
\hline$L(\mu \mathrm{m})$ & (Ref. 51-53) & 1 & 0.25 & 1 \\
\hline$a\left(\AA^{2}\right)$ & & $0.5 \pm 0.6$ & $10 \pm 5$ & $4.3 \pm 0.6$ \\
\hline$d S / d \Omega$ & & $0.1 \pm 0.2$ & $20 \pm 10$ & $6.5 \pm 0.8$ \\
\hline \multicolumn{5}{|c|}{$244 \mathrm{~nm}, 5.08 \mathrm{eV}$} \\
\hline$n$ & (Ref. 49 and 50) & 2.3 & 2.1 & 2.7 \\
\hline$L(\mu \mathrm{m})$ & (Ref. 51-53) & 1 & 0.1 & 1 \\
\hline$a\left(\AA^{2}\right)$ & & $0.4 \pm 0.6$ & $20 \pm 6$ & $4.7 \pm 0.6$ \\
\hline$d S / d \Omega$ & & $1 \pm 2$ & $1700 \pm 400$ & $160 \pm 20$ \\
\hline
\end{tabular}

$$
=\frac{8}{c^{4} V_{c} \mu} \frac{\omega_{s}^{3}}{\omega_{\mathrm{ph}}} \frac{N\left(\omega_{\mathrm{ph}}\right)+1}{(n+1)^{4}} P_{l} \Delta \Omega L a^{2},
$$

where $L$ is the scattering length and $n$ the index of refraction for a given excitation energy. Note that $R_{s}$ varies only as $\omega^{3}$, because the number of incoming photons is expressed as a laser power. In principle Eq. (6) can be used to directly measure the Raman susceptibility $a$. However, in practice this is difficult, because the sensitivity of the Raman setup (spectrometer and detector) depends strongly on the wavelength of the analyzed Raman light.

The spectrometer response can be measured using a standard light source as was done, e.g., by Calleja et al. ${ }^{46}$ with synchrotron radiation. The second, much easier, method is to normalize the measured integrated Raman intensity $I$ to the Raman intensity $I_{d}$ of a reference material obtained under exactly the same experimental conditions. We used diamond as a reference. The Raman susceptibility $a$ of boron nitride with respect to the known susceptibility of diamond $a_{d}$ can be found from Eq. (6): ${ }^{37,47,48}$

$$
\begin{aligned}
a= & {\left[\frac{V_{c} \mu}{V_{c, d} \mu_{d}} \frac{L_{d}}{L} \frac{(1+n)^{4}}{\left(1+n_{d}\right)^{4}} \frac{\left(\omega_{l}-\omega_{\mathrm{ph}, d}\right)^{3}}{\left(\omega_{l}-\omega_{\mathrm{ph}}\right)^{3}} \frac{\omega_{\mathrm{ph}}}{\omega_{\mathrm{ph}, d}}\right.} \\
& \left.\times \frac{N\left(\omega_{\mathrm{ph}, d}\right)+1}{N\left(\omega_{\mathrm{ph}}\right)+1} \frac{I}{I_{d}}\right]^{1 / 2} a_{d} .
\end{aligned}
$$

Variables without an additional subscript refer to the material under study, variables with a subscript $d$ to diamond. In deriving Eq. (7) we used the fact that the experimental setup and the laser intensity were the same for the $\mathrm{BN}$ and the diamond measurements.

The scattering length $L$ is given either by the sample thickness, or the focal length of the microscope objective, or by $L=1 / 2 \alpha$ ( $\alpha$ is the absorption coefficient), whichever is smallest. From the published absorption coefficients the scattering length in cubic $\mathrm{BN}$ and diamond is limited by the focal length of the microscope at our excitation energies. ${ }^{45,49,51,52}$ In hexagonal boron nitride, however, the absorption coefficient is on the order of $10^{4} \mathrm{~cm}^{-1}$ even for visible photon energies. ${ }^{53,54}$ The scattering length in this material is thus limited by the optical penetration depth, see Table II.

The Raman susceptibility for cubic and hexagonal boron nitride at 2.41 and $5.08 \mathrm{eV}$ measured by sample substitution are given in Table II. For convenience we also list the Raman scattering efficiency at both excitation energies, which is a better approximation to the scattering intensity observed in a Raman experiment. For cubic BN the Raman cross section found by sample substitution is approximately a factor of three smaller than obtained from the LO-TO ratio. Note, however, that the error bars are quite large. Also, measurements of the Raman susceptibility using different methods often vary quite strongly. ${ }^{37,48}$ The smaller value of $a$ measured by sample substitution than from the LO-TO ratio may have two main sources: the electro-optical coefficient of cubic BN is much smaller than predicted theoretically and/or the absorption coefficient of our sample is larger than in the measurements reported by Stenzel et al. ${ }^{50}$ and Onodera et $a l .{ }^{45} \mathrm{An}$ experimental determination of the electro-optical coefficient of cubic BN and other nitrides would be very desirable to obtain definite values for the Raman efficiency in the group III nitrides.

\section{Raman resonances in boron nitride}

The nonresonant Raman susceptibility as measured in the visible in cubic and hexagonal $\mathrm{BN}$ are similar to other wide band-gap materials (see Cardona ${ }^{37}$ for a compilation of results). In particular, the Raman susceptibility of the TO pho- 
non incubic GaN is $5 \AA^{2}$ (Ref. 48), i.e., in between the cubic and hexagonal BN values. When the excitation energy approaches the fundamental band gap of a material, the Raman susceptibility normally increases by orders of magnitude. We find, however, to first approximation a constant susceptibility in cubic and hexagonal boron nitride when using visible and UV excitation energies. The absence of resonance in cubic $\mathrm{BN}$ and the weak enhancement in hexagonal $\mathrm{BN}$ at $5.08 \mathrm{eV}$ can imply that our excitation energy is still small compared to the band gap. Another possibility is that no resonance occurs at the band gap of $\mathrm{BN}$ as was found at the direct gap in diamond. ${ }^{46}$

Cubic boron nitride has an indirect gap between 6.0 and $6.4 \mathrm{eV}$ (Ref. 45). Raman resonances at indirect gaps are often weak, because of the additional phonons necessary for the two optical transitions in the scattering process. The indirect resonance is thus higher order in pertubation theory than a resonance at a direct gap. Ralston et al. ${ }^{55}$ showed that at an indirect gap the resonant enhancement is reduced by a factor $\left[\mathcal{M}_{X} /\left(\hbar \omega_{d}-\hbar \omega_{l}\right)\right]^{2}$, where $\mathcal{M}_{X}$ is the electron-phonon matrix element for the phonons involved in the indirect optical transitions ( $X$ point phonons in cubic $\mathrm{BN}$ ), $\hbar \omega_{d}$ is the energy of the direct band gap, and $\hbar \omega_{l}$ the laser energy.

To see whether the indirect nature of the fundamental gap in cubic BN explains the constant Raman cross section obtained in our measurements we estimate the ratio of the Raman susceptibilities at a laser energy $\hbar \omega_{\mathrm{vis}} \approx 2.5 \mathrm{eV}$ and $\hbar \omega_{\mathrm{UV}} \approx 5 \mathrm{eV}$ following Ralston et al. ${ }^{55}$ For an order-ofmagnitude estimate we assume for cubic boron nitride $\mathcal{M}_{X}$ $\approx 1 \mathrm{eV}$ as is typically found in semiconductors. The direct band gap of cubic BN is $\hbar \omega_{d} \approx 10 \mathrm{eV} \cdot{ }^{56}$ For visible excitation the contribution from the indirect band gap can safely be neglected, because the crystal is transparent and all intermediate electronic states are virtual states. If the laser is in resonance with the indirect gap at $5 \mathrm{eV}$ we obtain a ratio between the Raman susceptibilities of cubic $\mathrm{BN}^{37,47,55}$

$$
\begin{aligned}
\frac{a\left(\omega_{\mathrm{vis}}\right)}{a\left(\omega_{\mathrm{UV}}\right)}=\frac{\omega_{\mathrm{UV}}}{\omega_{\mathrm{vis}}} \frac{\left|W_{F I}\left(\omega_{\mathrm{vis}}\right)\right|}{\left|W_{F I}\left(\omega_{\mathrm{UV}}\right)\right|} & \propto \frac{2}{\mathcal{M}_{X}^{2}} \frac{\left(\omega_{\mathrm{UV}}-\omega_{\mathrm{id}}\right)^{2}\left(\omega_{\mathrm{UV}}-\omega_{d}\right)^{2}}{\left(\omega_{\mathrm{vis}}-\omega_{d}\right)^{2}} \\
& \approx 1 .
\end{aligned}
$$

$\left|W_{F I}\right|$ is the Raman matrix element in the microscopic model of Raman scattering, see Trallero-Giner et al. ${ }^{47}$ for details; $\hbar \omega_{\mathrm{id}} \approx 6 \mathrm{eV}$ is the energy of the indirect gap. ${ }^{45}$ In agreement with our measurements we find a ratio of one between the visible and UV Raman susceptibilities. The cross section of cubic boron nitride is thus constant up to $5.4 \mathrm{eV}$ because of the indirect band gap of cubic BN and the very large direct gap at the $\Gamma$ point.

The situation is different for hexagonal BN. Hexagonal boron nitride is a direct semiconductor with $E_{g}=5.2 \mathrm{eV}$ at the $H$ point of the Brillouin zone. ${ }^{53}$ We thus expect a resonance when the laser at $5.08 \mathrm{eV}$ approaches the gap. To understand the nearly constant Raman susceptibility in our experiment we consider the macroscopic theory of Raman scattering. Within this model the resonant Raman susceptibility $a$ arising from two-band terms is proportional to the derivative of the susceptibility with respect to the photon frequency $\left|\chi^{\prime}\right|=|d \chi / d \omega|$. In Fig. 2 we plot the theoretically

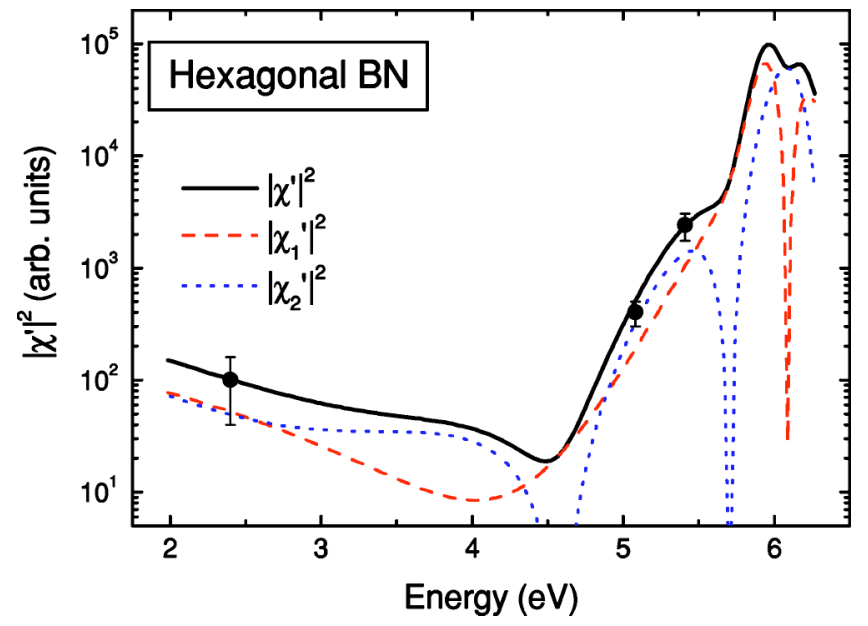

FIG. 2. (Color online) Theoretical dependence of the Raman cross section in hexagonal $\mathrm{BN}$ on laser energy together with the three measured points. $\left|\chi^{\prime}\right|^{2}=|d \chi / d \omega|^{2}$ (full black line) was separated into its real $\left|\chi_{1}^{\prime}\right|^{2}$ (red, dashed) and imaginary $\left|\chi_{2}^{\prime}\right|^{2}$ (blue, dotted) part. The theoretical and experimental data were multiplied by constant factors to match at $2.41 \mathrm{eV}$. The derivatives were calculated from the dielectric function of hexagonal $\mathrm{BN}$ measured by Hoffman et al. (Ref. 53).

expected resonant enhancement of hexagonal BN by the full black line. The curve was obtained from the dielectric function measured by Hoffman et al..$^{53}$ The energies were shifted by $\hbar \omega_{\mathrm{ph}} / 2=0.08 \mathrm{eV}$ to higher energies as is normally done to account for incoming and outgoing resonance terms in the Raman process. ${ }^{57}$

According to Fig. 2 the Raman susceptibility of hexagonal BN first decreases between 2 and $4.5 \mathrm{eV}$. It then starts to increase when the fundamental gap at $5.2 \mathrm{eV}$ is approached and, finally, peaks just below $6 \mathrm{eV}$. This maximum corresponds to the optical transitions at the $M$ point of the Brillouin zone. These transitions give rise to a large peak in the measured absorption coefficient. ${ }^{53}$ Also shown in Fig. 2 are the squares of the Raman susceptibility at three excitation energies. The theoretical curves were normalized to the experimental susceptibility at $2.41 \mathrm{eV}$. The data points at 2.41 and $5.08 \mathrm{eV}$ were taken from Table II. At $5.41 \mathrm{eV}$ we normalized the Raman intensity of hexagonal $\mathrm{BN}$ to the TO phonon of cubic BN, since we found a constant susceptibility in cubic $\mathrm{BN}$ from the LO-TO ratio and the theoretical discussion presented above $\left(a=50 \AA^{2}\right.$ at $5.41 \mathrm{eV}, d S / d \Omega=1.4$ $\left.\times 10^{-1} \mathrm{~m}^{-1} \mathrm{Sr}^{-1}\right)$.

The agreement between the measured data points and the theoretical curve in Fig. 2 is excellent. This is somewhat surprising, because the absorption in hexagonal $\mathrm{BN}$ below $5 \mathrm{eV}$ is due to impurity and defect states in the band gap. ${ }^{53}$ The comparison between $|d \chi / d \omega|$ and the experimental susceptibility is rather justified a posteriori. Nevertheless, according to Fig. 2 the almost constant Raman susceptibility at 2.41 and $5.08 \mathrm{eV}$ is due to the decreasing cross section in the visible and the near UV. It would be very interesting to measure the Raman scattering efficiency of hexagonal $\mathrm{BN}$ in the energy range between 3 and $4.5 \mathrm{eV}$ to see whether the predicted decrease is found experimentally as well.

In a Raman experiment the measured intensity is proportional to the scattering efficiency $d S / d \Omega$, see Eq. (5); the 
values are included in Tables I and II. The scattering efficiency of hexagonal $\mathrm{BN}$ is two to three orders of magnitude larger than the efficiency of the cubic phase. Note also the increase in $d S / d \Omega$ in the UV by a factor of $10-100$ when compared to the visible. This enhancement comes mainly from the $\omega^{4}$ dependence of the Raman scattering efficiency. ${ }^{37}$ The large Raman cross section of hexagonal BN suggests that UV Raman scattering is extremely powerful in detecting the hexagonal minority phase in nominally cubic boron nitride films. A volume ratio of $1: 10^{3}$ between cubic and hexagonal $\mathrm{BN}$ gives rise to the same scattering intensity for the 1055 cubic TO and the $1364 \mathrm{~cm}^{-1} E_{2 g}$ hexagonal phonon. A ratio of $10^{4}$ should be easily detected. In infrared spectroscopy the corresponding ratio is very close to $1,{ }^{58}$ i.e., infrared absorption is much less sensitive for detecting hexagonal BN than UV Raman scattering. In view of the high sensitivity of UV Raman scattering to the hexagonal BN phase, it would be very interesting to measure UV Raman spectra on the high-quality cubic BN films that were grown expitaxially on diamond. ${ }^{10}$

\section{SECOND-ORDER RAMAN SCATTERING}

With UV excitation the Raman scattering efficiency of cubic and hexagonal $\mathrm{BN}$ was large enough to observe welldefined second-order Raman spectra. Moreover, the broad background typical for visible excitation was absent when using UV excitation energies. To best of our knowledge this is the first report of second-order Raman scattering in boron nitride. The second-order spectra are dominated by overtones of the phonon branches and thus provide a rigid test for theoretical lattice dynamics. In the following we first discuss cubic boron nitride, where we found an excellent agreement between $a b$ initio calculations and the second-order Raman spectrum. The spectrum of hexagonal BN is strongly structured. It shows sharp peaks due to the layered hexagonalstructure of this boron nitride form. While most peaks agree well between theory and experiment, we observed strong deviations for the nitrogen-related zone-boundary vibrations.

\section{A. Cubic boron nitride}

The phonon dispersion of cubic BN was calculated by a number of groups; ${ }^{28,29,59}$ most elaborate was the study by Karch and Bechstedt. ${ }^{59}$ They pointed out that the phonon dispersion and phonon density of states in cubic BN resembles the one of diamond. In the low-energy range the acoustic branches are strongly dispersive. In contrast to most other III-V compounds cubic boron nitride therefore has an unstructured and flat phonon density of states in the acoustic range. The density of states in the optical ranges is well separated into features coming from the transverse (between 1700 and $2100 \mathrm{~cm}^{-1}$ ) and longitudinal (2100-2700 $\mathrm{cm}^{-1}$ ) optical branches. The calculated phonon density of states by Karch and Bechstedt ${ }^{59}$ is reproduced in Fig. 3 by the dashed line. Also shown in the figure is the second-order Raman spectrum of cubic BN. Only the range of the optical modes is displayed, since the second-order signal in the acoustic range is indeed without remarkable structures as predicted by the

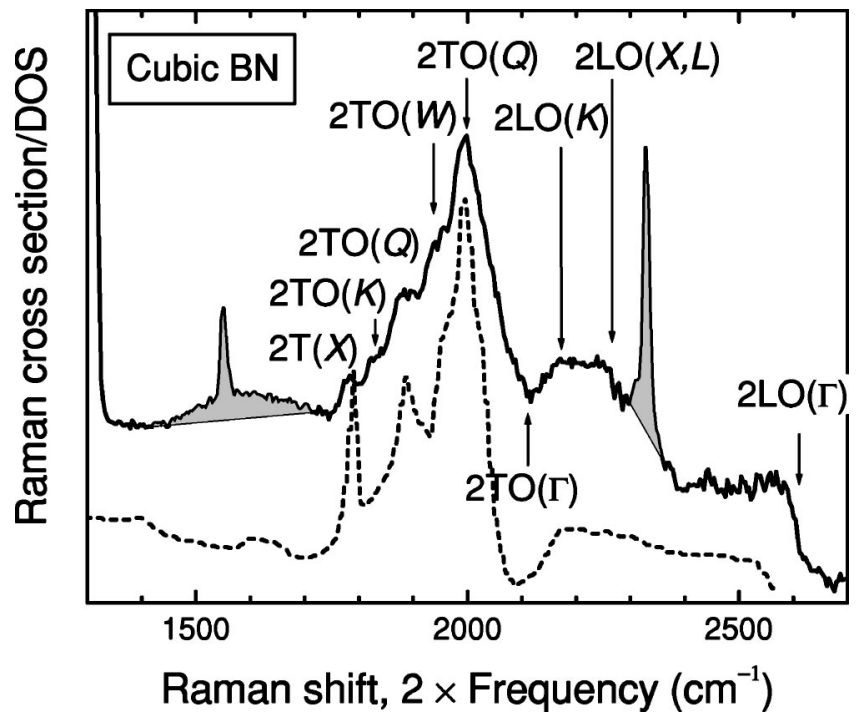

FIG. 3. Full line: Second-order Raman spectrum of cubic boron nitride excited with $229 \mathrm{~nm}$. The gray-shaded areas correspond to the $\mathrm{O}_{2}\left(1556 \mathrm{~cm}^{-1}\right)$ and $\mathrm{N}_{2}\left(2332 \mathrm{~cm}^{-1}\right)$ air-related lines and background from the setup. Broken line: $A b$ initio calculation of the phonon density of states by Karch and Bechstedt (Ref. 59); the frequencies were multiplied by two. Peaks and shoulders in the spectrum were assigned to high-symmetry points and lines in the boron nitride Brillouin zone, see also Table III. All structures are well explained as overtones of the phonon density of states. Compared to Fig. 1(a) the $y$ scale is expanded by a factor of 20 .

$a b$ initio calculation.

As can be seen in Fig. 3 the agreement between theory and experiment is excellent. In particular, the transverse phonons give rise to a number of peaks in the second-order Raman spectrum that match very well with the calculated density of states. Table III summarizes the measured and calculated frequencies in cubic BN; note also the excellent agreement between the frequencies of the first-order Raman signal and the corresponding features in the second-order spectrum.

TABLE III. Experimentally observed frequencies in the secondorder Raman spectrum of cubic boron nitride assigned to phonon branches and critical points in the Brillouin zone. The theoretical values were taken from the ab-initio calculation by Karch and Bechstedt (Ref. 59). All numbers were rounded to $5 \mathrm{~cm}^{-1}$.

\begin{tabular}{ccc}
\hline \hline experiment $\left(\mathrm{cm}^{-1}\right)$ & branch & Theory $\left(\mathrm{cm}^{-1}\right)$ \\
\hline 900 & $\mathrm{TO}(X)$ & 900 \\
915 & $\mathrm{TO}(K)$ & 910 \\
940 & $\mathrm{TO}(Q)$ & 945 \\
970 & $\mathrm{TO}(W)$ & 965 \\
1000 & $\mathrm{TO}(Q)$ & 1000 \\
1055 & $\mathrm{TO}(\Gamma)$ & 1035 \\
1085 & $\mathrm{LO}(K)$ & 1075 \\
1135 & $\mathrm{LO}(L)$ & 1140 \\
1135 & $\mathrm{LO}(X)$ & 1145 \\
1305 & $\mathrm{LO}(\Gamma)$ & 1285 \\
\hline \hline
\end{tabular}




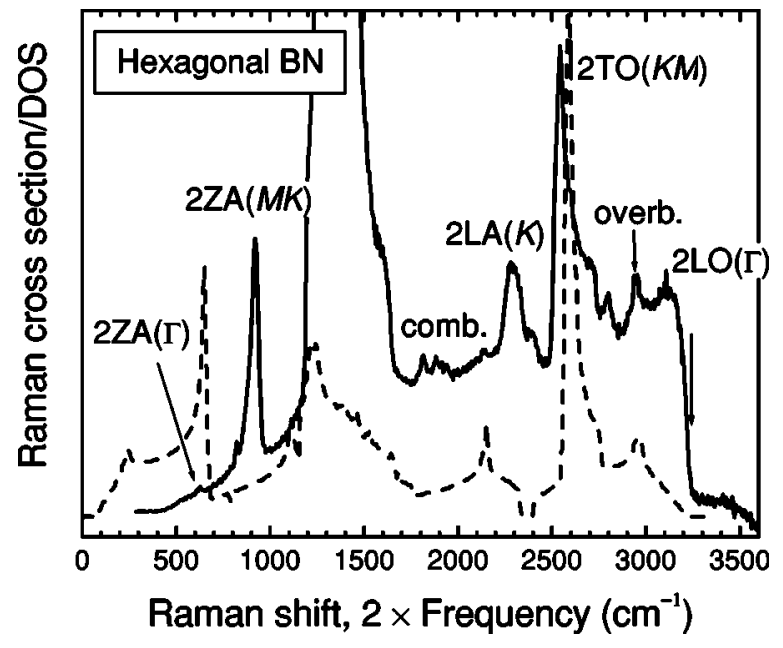

FIG. 4. Second-order Raman scattering in hexagonal boron nitride. The full line is the experimental spectrum. The broken line is the phonon density of states after Kern et al. (Ref. 28); the frequencies were multiplied by 2 . For the most prominent features in the second-order spectrum the assignment is indicated, see Table IV for the measured frequencies and Fig. 5 for the labeling of the branches. Compared to Fig. 1(b) the $y$ scale is expanded by a factor of 300 .

Second-order Raman scattering by the longitudinal optical phonons when compared to the transverse modes is somewhat larger in intensity than expected from the phonon density of states, see Fig. 3. Also, the peaks and dips that correspond to Raman scattering by LO phonons from highsymmetry points in the Brillouin zone are better seen in the experiment than in the calculations. The LO phonons belong to the totally symmetric representation in most parts of the Brillouin zone. Totally symmetric modes often have a particularly large electron-phonon interaction. This explains the higher intensities of the LO related features in the Raman spectrum when compared to the phonon density of states.

Judging from our second-order spectra, the calculation by Karch and Bechstedt ${ }^{59}$ gives a most reliable description of the phonon dispersion in cubic boron nitride. Our experiments confirm the strong dispersion of the acoustic phonons. The transverse and longitudinal optical branches of cubic BN do not cross along $\Gamma-L$ as in many other III-V semiconductors. ${ }^{59}$ This results in the two separate frequency ranges for the $\mathrm{LO}$ and $\mathrm{TO}$ branches, which we found experimentally as well.

\section{B. Hexagonal boron nitride}

In Fig. 4 we show the second-order Raman spectrum of hexagonal boron nitride (full line). As for cubic boron nitride several features from higher-order Raman scattering are clearly observed in the spectra in our UV Raman measurement. The broken line in Fig. 4 is the ab initio phonon density of states by Kern et al. $;^{28}$ similar results were obtained by $\mathrm{Yu}$ et al. ${ }^{29}$ and also in calculations of a single sheet of hexagonal BN. ${ }^{25,26}$ The agreement between the calculated phonon density of states and the second-order Raman measurements is very good, especially, at high phonon energy.

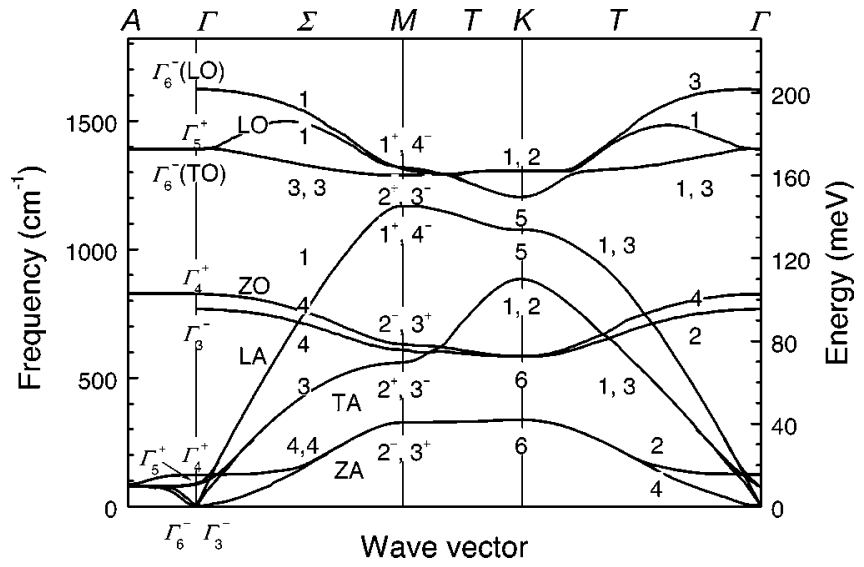

FIG. 5. Phonon dispersion of hexagonal boron nitride calculated by Kern et al. (Ref. 28). The phonon branches at the high-symmetry points and lines in the Brillouin zone were assigned to the irreducible representations of the BN group. The numbers correspond to the subscript of the irreducible representation and the $+/-$ sign to the superscript. For example, the two lowest branches at the $M$ point labeled $2^{-}$and $3^{+}$belong to the $M_{2}^{-}$and $M_{3}^{+}$representation. The degeneracy of these two phonons is accidental. At the highsymmetry points all phonon overtones and the following combinations give rise to in-plane polarized second-order Raman scattering $\Gamma_{1}^{ \pm} \otimes \Gamma_{5}^{ \pm}, \Gamma_{2}^{ \pm} \otimes \Gamma_{6}^{ \pm}, M_{3}^{ \pm} \otimes M_{4}^{ \pm}, M_{1}^{ \pm} \otimes M_{2}^{ \pm}, K_{1,2} \otimes K_{5}$, and $K_{3,4} \otimes K_{6}$.

The most prominent maxima in the density of states have their counterparts in the measured spectrum. In the following we discuss the different parts of the second-order spectrum starting from the highest energies, where the agreement between theory and experiment is best.

For the following discussion we reproduced the calculated phonon dispersion by Kern et al. ${ }^{28}$ in Fig. 5. We assigned all the phonon branches by their irreducible representations in the interior of the Brillouin zone.

The extremely sharp feature at $2540 \mathrm{~cm}^{-1}$ in Fig. 4 comes from the overtones of the flat transverse optical branch between the $M$ and $K$ point of the BN Brillouin zone. This peak is labeled $2 \mathrm{TO}(K M)$ in Fig. 4, all frequencies are compiled in Table IV. The full width at half maximum of the $2 \mathrm{TO}(K M)$ peak is $80 \mathrm{~cm}^{-1}$, implying a band width of $40 \mathrm{~cm}^{-1}$ between $M$ and $K$. The frequency of the transverse optical band in boron nitride thus varies by only $7 \%$ between its maximum at the $\Gamma$ point and the minimum at $M$. In graphite-a structurally related compound - the corresponding value is $20 \% .^{60}$ The reason for the large differences in the phonon dispersion of hexagonal $\mathrm{BN}$ and graphite is that graphite is a semimetal. This gives rise to Kohn anomalies and a strong softening of some phonon modes in graphite. ${ }^{61}$

Above $2540 \mathrm{~cm}^{-1}$ the phonon density of states in Fig. 4 sharply decreases and the step at $2730 \mathrm{~cm}^{-1}$ marks twice the frequency of the Raman-active $\Gamma$ point mode. The secondorder value for the $\Gamma$-point frequency $\left(1365 \mathrm{~cm}^{-1}\right)$ is in excellent agreement with the first-order Raman measurement discussed in Sec. III.

The peak at $2940 \mathrm{~cm}^{-1}$ (labeled overb. in Fig. 4) is associated with the overbending of the in-plane longitudinal optical phonon. The highest frequency of the $\Gamma_{5}^{+}$or $E_{2 g}$ highenergy phonon is not at the $\Gamma$ point, but at approximately $1 / 2$ 
TABLE IV. Experimentally observed frequencies assigned to critical points in the hexagonal boron nitride Brillouin zone. For the overtones the measured phonon energies were divided by 2; for the combinations the measured values are given. The theoretical phonon frequencies are taken from Refs. 28 and 29. All frequencies were rounded to $5 \mathrm{~cm}^{-1}$; fo stands for first-order measurements.

\begin{tabular}{|c|c|c|c|}
\hline Experiment $\left(\mathrm{cm}^{-1}\right)$ & Branch & Symmetry & Theory $\left(\mathrm{cm}^{-1}\right)$ \\
\hline \multicolumn{4}{|c|}{ Overtones peaks } \\
\hline 310 & $\mathrm{ZA}(\Gamma)$ & $\Gamma_{4}^{+}$ & 125 \\
\hline 460 & $\mathrm{ZA}(M-K)$ & $M_{2}^{+}, M_{3}^{-}, K_{6}$ & 330 \\
\hline 1145 & $\mathrm{LA}(K)$ & $K_{5}$ & 1075 \\
\hline 1270 & $\mathrm{TO}(M-K)$ & $M_{2}^{+}, M 3^{-}, K_{1}, K_{2}$ & 1290 \\
\hline 1364(fo) & $\mathrm{TO}, \mathrm{LO}(\Gamma)$ & $\Gamma_{5}^{+}$ & 1380 \\
\hline \multicolumn{4}{|l|}{1400} \\
\hline 1470 & LO (overbending) & $T_{3}, \Sigma_{1}$ & 1490 \\
\hline \multicolumn{4}{|c|}{ Overtones flanks } \\
\hline 820 & $\mathrm{ZO}$ & $\Gamma_{3}^{-}, \Gamma_{4}^{+}$ & 830 \\
\hline 1210 & $\mathrm{LA}(M)$ & $M_{1}^{+}, M_{4}^{-}$ & 1160 \\
\hline 1365 & $\mathrm{TO}, \mathrm{LO}(\Gamma)$ & $\Gamma_{5}^{+}$ & 1380 \\
\hline 1605 & $\mathrm{LO}(\Gamma)$ & $\Gamma_{6}^{-}$ & 1610 \\
\hline \multicolumn{4}{|c|}{ Combination peaks } \\
\hline 1810 & $\mathrm{TA}+\mathrm{LA}(M)$ & $M_{1}^{+} \otimes M_{2}^{+}, M_{3}^{-} \otimes M_{4}^{-}$ & 1720 \\
\hline 1880 & $\mathrm{TA}+\mathrm{TO}(M)$ & $M_{2}^{+} \otimes M_{2}^{+}, M_{3}^{-} \otimes M_{3}^{-}$ & 1840 \\
\hline 1920 & $\mathrm{TA}+\mathrm{LA}(K)$ & $K_{1} \otimes K_{5}$ & 1960 \\
\hline 2140 & $\mathrm{TA}+\mathrm{TO}(K)$ & $K_{1} \otimes K_{1}, K_{2} \otimes K_{2}$ & 2185 \\
\hline
\end{tabular}

between $\Gamma$ and the boundary of the Brillouin zone, see Fig. 5. ${ }^{28,29}$ Overbending manifests itself in a second-order signal that is higher than twice the first-order frequency. So far graphite and diamond were thought to be the prototypes for this anomaly in the phonon dispersion. The overbending in graphite amounts $30 \mathrm{~cm}^{-1}$, but only $2 \mathrm{~cm}^{-1}$ in diamond. ${ }^{60,62-65}$ In hexagonal boron nitride we find an overbending of $100 \mathrm{~cm}^{-1}$, see Table IV. This is much bigger than in any carbon compound.

There is no obvious assignment for the peak at $2800 \mathrm{~cm}^{-1}$, i.e., between the $2 \mathrm{TO}(K M)$ and the peak coming from the LO overbending in Fig. 4. It is too high in frequency to be an overtone of the optical $\Gamma$-point vibration. Yu et $a{ }^{29}$ predicted the overbending along the $\Gamma M$ and $\Gamma K$ direction to be different, which could explain the appearance of the additional maximum. However, more theoretical and experimental work is necessary to clarify this point. Finally, the sharp drop of the scattering intensity at $3210 \mathrm{~cm}^{-1}$ marks the overtone of the antisymmetric LO phonon and the highenergy end of the phonon density of states, see $2 \mathrm{LO}(\Gamma)$ in Fig. 4. The corresponding first-order frequency, $1605 \mathrm{~cm}^{-1}$, is in excellent agreement with infrared measurements $\left(1610 \mathrm{~cm}^{-1}\right)$ and $a b$ initio calculations. ${ }^{28-30,66}$

Geick et al $^{30}$ reported a second-order peak in the infrared spectra of hexagonal boron nitride at $\approx 2600 \mathrm{~cm}^{-1}$, i.e., in the energy range discussed so far. The feature was much broader than in the Raman spectra in Fig. 4. Unfortunately, the relative strength of the $2600 \mathrm{~cm}^{-1}$ peak is not given in Ref. 30, but it seems much weaker than expected from the phonon density of states. According to us the main contribution to the Raman signal between 2550 and $3000 \mathrm{~cm}^{-1}$ comes from the TO branch at $K$ and $M\left(M_{2}^{+}, M_{3}^{-}, K_{1}\right.$, and $K_{2}$ symmetry) and weaker signals from the longitudinal optical branches $\left(M_{1}^{+}, M_{4}^{-}, K_{5}\right.$ representations at the high symmetry points), see the symmetry assignment in Fig. 5. For these representations only combinations are infrared active, except for the overtone of the LO at $K .^{67,68}$ The $K_{5}$ overtone and some of the possible combinations correspond to the in-plane infrared active representation. Indeed the in-plane signal reported by Geick et al. $^{30}$ is stronger than the signal with out-of-plane polarization. The selection rules, which forbid absorption by the TO overtones, thus explain why the infrared absorption is much weaker than the Raman signal at very high frequencies.

We now focus to the other energy ranges of the secondorder Raman spectrum. The calculated phonon density of states shows three main peaks, which have their counterparts in the experimental spectrum. Unfortunately, the region around $1400 \mathrm{~cm}^{-1}$ is shadowed by the strong first-order Raman line. Note that in hexagonal boron nitride it is not possible to suppress first-order Raman scattering by selection rules. The $\Gamma_{5}^{+}$phonon is allowed in parallel as well as crossed polarization of the incoming and outgoing light. ${ }^{67,68}$ Nevertheless, the first order Raman peak has a shoulder at high energies and one at the low-energy side. This scattering comes from the overtones of the transverse acoustic and outof-plane optical modes; we tentatively assign the highenergy flank to the $\mathrm{ZO} \Gamma$ point overtone.

The two peaks at 920 and $2290 \mathrm{~cm}^{-1}$ in the experimental spectrum, 2ZA $(M K)$ and $2 \mathrm{LA}(K)$ in Fig. 4, bear a striking similarity to the shape of the calculated phonon density of 
states. Note also the small peak labeled $2 \mathrm{ZA}(\Gamma)$ and the step at the high-energy side of the $2290 \mathrm{~cm}^{-1}$ peak. The calculated phonon frequencies, however, are smaller by 40 and $7 \%$ than observed experimentally. ${ }^{28,29}$ Let us assume that the singularities in the second-order spectrum indeed correspond to the singularities in the calculated density of states. From the symmetries of the phonon species given in Table IV we expect the same overtones in the infrared spectrum for light polarized along the hexagonal $\mathrm{BN}$ planes; for perpendicular polarization the overtones (and the combinations) are forbidden by selection rules. This is in excellent agreement with the $920 \mathrm{~cm}^{-1}$ absorption peak reported by Geick et al. ${ }^{30}$ for $E \perp \boldsymbol{a}_{3}$; for light polarized parallel to $\boldsymbol{a}_{3}$ no infrared absorption was detected. Unfortunately, for the high-energy feature at $2290 \mathrm{~cm}^{-1}$ the absorption was not measured in the infrared experiment.

Let us discuss other possible assignments of the 920 and $2290 \mathrm{~cm}^{-1}$ features. From the $a b$ initio frequencies and the symmetries of the eigenvectors, the $920 \mathrm{~cm}^{-1}$ peak can also originate from a combination of the optical and acoustic outof-plane phonons ZA $+Z O$, while the $2290 \mathrm{~cm}^{-1}$ band can be a combination of the longitudinal optical and acoustic inplane branch LA+LO, see Fig. 5. ${ }^{25,26,28,29,66}$ This assignment implies, however, that a combination of two phonon branches is very intense, whereas the corresponding overtones with a high density of states are absent. This scenario, however, contradicts the well-known finding that secondorder Raman spectra are normally dominated by overtones, ${ }^{69,70}$ although we cannot rule out this possibility on the basis of the selection rules.

A phonon peak at $920 \mathrm{~cm}^{-1}$ was observed in hexagonal BN by infrared spectroscopy and in surface enhanced Raman scattering (SERS) on cubic BN..$^{30,71,72}$ Geick et al..$^{30}$ assigned the infrared feature to a second-order phonon of hexagonal boron nitride. Zedlitz et al. ${ }^{71}$ and Zhang et al. ${ }^{72}$ however, argued that the peak at $920 \mathrm{~cm}^{-1}$ is due to the rocking vibration of a boron-hydrogen complex. The low-energy SERS spectrum is remarkably similar to the $2 \mathrm{ZA}(\Gamma)$ and $2 \mathrm{ZA}(M K)$ peaks in Fig. 4. The high-energy modes that Zhang ${ }^{72}$ assigned to $\mathrm{NH}_{2}$ scissoring and $\mathrm{BH}$ stretching vibrations, on the other hand, all have their counterparts as overtones in the phonon density of states of cubic boron nitride. ${ }^{28,29}$ Most of the SERS modes of the hydrogen treated surface can thus straightforwardly be assigned to an enhancement of the bulk phonons by silver particles and either surface vibrations of nitrogen complexes or the presence of hexagonal boron nitride in the sample. The large Raman cross section of hexagonal BN makes the last explanation very likely. The $920 \mathrm{~cm}^{-1}$ peak appears in infrared reflectivity measurements only for in-plane polarized light and is also Raman active. ${ }^{30}$ Within the $\mathrm{BH}$ rocking explanation this implies that the $\mathrm{BH}_{2}$ complexes are strictly aligned within the $\mathrm{BN}_{2}$ planes, which, again, is rather unexpected.

We tentatively assign the 920 and $2290 \mathrm{~cm}^{-1}$ peaks to the predicted singularities in the phonon density of states. This implies that the shape of the phonon dispersion was correctly predicted by the $a b$ initio calculations. ${ }^{28,29}$ The calculated frequencies, however, are too small. Interestingly, both peaks would originate from eigenvectors where only the nitrogen atoms move. ${ }^{25}$ The low-energy singularity involves the out- of-plane acoustic phonon of the nitrogen atoms; the highenergy singularity the LA branch. The purely boron-related vibrations are not observable experimentally, because they are either masked by the first-order Raman peak (ZA branch) or do not give rise to a singularity in the phonon density of states (LA branch).

The experiment to perform in order to validate the possible assignment of these peaks to nitrogen overtones would be isotope substitution. Substituting ${ }^{14} \mathrm{~N}(99.63 \%$ natural abundance) with the other stable nitrogen isotope ${ }^{15} \mathrm{~N}$ the 920 and $2290 \mathrm{~cm}^{-1}$ peaks would shift by 30 and $80 \mathrm{~cm}^{-1}$ to smaller frequencies, respectively. If the two peaks are due to combinations, the shifts would be much smaller (10 and $40 \mathrm{~cm}^{-1}$ ); for the $\mathrm{BH}_{2}$ rocking vibration obviously the phonon frequencies must remain unchanged.

The small peak at $620 \mathrm{~cm}^{-1}$ in Fig. 4. $[2 \mathrm{TA}(\Gamma)]$ is an overtone of the rigid layer vibration along the $\boldsymbol{a}_{3}$ axis. The energy of this mode, $310 \mathrm{~cm}^{-1}$, is more than twice as much as predicted theoretically. ${ }^{28,29}$ Phonons with very low energy are difficult to calculate and to some extent deviations are expected. The significance of this particular phonon is that the high-pressure phase transition from hexagonal to wurzite boron nitride involves a decrease of the $\boldsymbol{a}_{3}$ lattice constant.

Finally, the second-order Raman signal in Fig. 4 shows some small peaks that are not related to overtones (labeled "comb"). We assign the four peaks to scattering by combinations from the $M$ and $K$ critical points, see Table IV and Fig. 5. From the frequencies of the experimentally observed peaks one of the phonons must be of high energy, i.e., belong to the LO, TO, or LA branch. When considering the selection rules for second-order Raman scattering the only possible second phonon is the TA mode. At first sight, the agreement between the experimentally observed and the predicted frequencies is not so good, see Table IV. However, if we take the LA and TO frequencies as obtained from second-order Raman scattering in this paper instead of the $a b$ initio values, we get consistent results with $\mathrm{TA}(M) \approx 600$ and $\mathrm{TA}(K)$ $\approx 800 \mathrm{~cm}^{-1}$. This agrees quite well with the calculated dispersion in Fig. 5; the corresponding frequencies are 560 and $890 \mathrm{~cm}^{-1}$, respectively.

\section{CONCLUSIONS}

We presented an in-depth study of the Raman spectrum of cubic and hexagonal boron nitride. We measured the absolute Raman efficiency in the visible and UV energy range. The nonresonant Raman cross section of boron nitride is similar to the cross section of $\mathrm{GaN}$ and other wide band-gap semiconductors. Hexagonal BN has a 100-1000 times larger scattering efficiency than the cubic form. Raman scattering is thus very powerful for measuring the volume fraction of hexagonal BN in thin boron-nitride films and to study boron nitride nanotubes.

The Raman susceptibility of cubic boron nitride is constant for all three excitation energies. The absence of resonance in cubic BN is due to the indirect band gap and the extremely high direct gap in this semiconductor. A Raman resonance is only expected for excitation energies close to the direct gap at $\Gamma$, i.e., $\approx 10 \mathrm{eV}$ excitation energy. In turn, 
this makes cubic boron nitride a good reference material for UV measurements of the absolute Raman efficiencies. Cubic $\mathrm{BN}$ is better suited for UV energies than the flourides used in the visible because of its high phonon frequency.

In hexagonal BN the Raman susceptibility at $5 \mathrm{eV}$ was twice as large as in the visible; at $5.4 \mathrm{eV}$ it was enhanced by a factor of 5. This marks the onset of the resonance in hexagonal BN. We find good agreement between the theoretical Raman susceptibility $|d \chi / d \omega|$ calculated from the dielectric function and our experimental cross sections. According to the theoretical data Raman scattering in hexagonal BN gets weaker between 2 and $4.5 \mathrm{eV}$ and then increases strongly with a maximum at $\approx 6 \mathrm{eV}$, i.e., at the energy of the optical transitions at the $M$ point of the hexagonal Brillouin zone.

Using UV excitation we measured second-order Raman scattering in cubic and hexagonal BN. The spectra are dominated by overtones. Comparing the second-order spectrum of cubic BN to $a b$ initio calculations of the phonon density of states we find excellent agreement between theory and experiment. The lattice dynamics of cubic BN are thus very well described by existing calculations. For hexagonal BN the agreement is less satisfactory. The in-plane optical vibrations are excellently described by published ab initio calculations. We assign the peak at $920 \mathrm{~cm}^{-1}$ to the overtone of the out-of-plane acoustical phonon between the $K$ and the $M$ point of the Brillouin zone or to a combination of two modes from acoustic and optical branches. We dismiss the assignment, often found in literature, of this peak to a BH rocking vibration.

Our measurements are a test for the many theoretical studies on the phonon dispersion of cubic and hexagonal boron nitride. We showed that UV Raman scattering is a very promising tool for characterizing thin boron nitride films and to find the volume ratio of the two boron nitride phases. This is of particular interest in view of the recent progress in growing high-quality cubic boron nitride films expitaxially on diamond.

\section{ACKNOWLEDGMENTS}

We wish to thank D. Bachtelder, A. Smith, and I. R. Mendieta of the University of Leeds and D. Wolverson of the University of Bath for access to their UV Raman facilities and kind hospitality in their laboratories. The cubic boronnitride samples were supplied by A. Sokolov and N. V. Novikov from the Bukul Institute of Superhard Materials NAUS in Kiev, Ukraine. A.C.F. acknowledges funding by the Royal Society. S.R. was supported by the Oppenheimer Fund and Newnham College.
${ }^{1}$ P. B. Mirkarimi, K. F. McCarty, and D. L. Mellin, Mater. Sci. Eng., R. 21, 47 (1997).

${ }^{2}$ W. Zhang, S. Matsumoto, Q. Li, I. Bello, and S. T. Lee, Adv. Funct. Mater. 12, 250 (2002).

${ }^{3}$ A. Catellani, M. Posternak, A. Baldereschi, and A. J. Frieman, Phys. Rev. B 36, 6105 (1987).

${ }^{4}$ G. Demazeau, Diamond Relat. Mater. 4, 284 (1995).

${ }^{5}$ S. Ulrich, J. Schwan, W. Donner, and H. Erhardt, Diamond Relat. Mater. 5, 548 (1996).

${ }^{6}$ M. P. D’Evelyn and T. Taniguchi, Diamond Relat. Mater. 8, 1522 (1992)

${ }^{7}$ D. J. Kester and R. Messier, J. Appl. Phys. 72, 504 (1992).

${ }^{8}$ W. M. Lau, D. Chen, Z. Song, N. S. McIntyre, Z. Deng, and R. M. Kwok, Surf. Interface Anal. 27, 698 (1999).

${ }^{9}$ T. Taniguchi and S. Yamaoka, J. Cryst. Growth 222, 549 (2001).

${ }^{10}$ X. W. Zhang, H.-G. Boyen, N. Deynaka, P. Ziemann, F. Banhart, and M. Schreck, Nat. Mater. 2, 312 (2004).

${ }^{11}$ W. Zhang, I. Bello, Y. Lifshitz, K. Chan, X. Meng, Y. Wu, C. Chan, and S. Lee, Adv. Mater. (Weinheim, Ger.) 16, 1405 (2004).

${ }^{12}$ W. Zhang, I. Bello, Y. Lifshitz, K. Chan, Y. Wu, C. Chan, X. Meng, and S. Lee, Appl. Phys. Lett. 85, 1344 (2004).

${ }^{13}$ N. G. Chopra, R. J. Luyken, K. Cherrey, V. H. Crespi, M. L. Cohen, S. G. Louie, and A. Zettl, Science 269, 966 (1995).

${ }^{14}$ A. Loiseau, F. Willaime, N. Demoncy, G. Hug, and H. Pascard, Phys. Rev. Lett. 76, 4737 (1996).

${ }^{15}$ D. Golberg, Y. Bando, M. Eremets, K. Takemura, K. Kurashima, and H. Yusa, Appl. Phys. Lett. 69, 2045 (1996).

${ }^{16}$ M. Terrones, W. K. Hsu, H. Terrones, J. P. Zhang, S. Ramos, J. P. Hare, R. Castillo, K. Prassides, A. K. Cheetham, H. W. Kroto, and D. R. M. Walton, Chem. Phys. Lett. 259, 568 (1996).

${ }^{17}$ S. Reich, C. Thomsen, and J. Maultzsch, Carbon Nanotubes: Basic Concepts and Physical Properties (Wiley-VCH, Berlin, 2004).

${ }^{18}$ R. Saito, T. Takeya, T. Kimura, G. Dresselhaus, and M. S. Dresselhaus, Phys. Rev. B 57, 4145 (1998).

${ }^{19}$ S. Reich, C. Thomsen, and P. Ordejón, Phys. Rev. B 65, 155411 (2002).

${ }^{20}$ A. Rubio, J. L. Corkill, and M. L. Cohen, Phys. Rev. B 49, 5081 (1994).

${ }^{21}$ X. Blase, A. Rubio, S. G. Louie, and M. L. Cohen, Europhys. Lett. 28, 335 (1994).

${ }^{22}$ X. Blase, A. Rubio, S. G. Louie, and M. L. Cohen, Phys. Rev. B 51, 6868 (1995).

${ }^{23}$ N. G. Chopra and A. Zettl, Solid State Commun. 105, 297 (1998).

${ }^{24}$ E. Hernández, C. Goze, P. Bernier, and A. Rubio, Phys. Rev. Lett. 80, 4502 (1998).

${ }^{25}$ D. Sánchez-Portal and E. Hernández, Phys. Rev. B 66, 235415 (2002).

${ }^{26}$ L. Wirtz, A. Rubio, R. A. de la Concha, and A. Loiseau, Phys. Rev. B 68, 045425 (2003).

${ }^{27}$ V. N. Popov, Phys. Rev. B 67, 085408 (2003).

${ }^{28}$ G. Kern, G. Kresse, and J. Hafner, Phys. Rev. B 59, 8551 (1999).

${ }^{29}$ W. J. Yu, W. M. Lau, S. P. Chan, Z. F. Liu, and Q. Q. Zheng, Phys. Rev. B 67, 014108 (2003).

${ }^{30}$ R. Geick, C. H. Perry, and G. Rupprecht, Phys. Rev. 146, 543 (1966).

${ }^{31}$ R. J. Nemanich, S. A. Solin, and R. M. Martin, Phys. Rev. B 23, 6348 (1981). 
${ }^{32}$ J. A. Sanjurjo, E. López-Cruz, P. Vogl, and M. Cardona, Phys. Rev. B 28, 4579 (1983).

${ }^{33}$ J. Liu, Y. K. Vohra, J. T. Tarvin, and S. S. Vagarali, Phys. Rev. B 51, 8591 (1995).

${ }^{34}$ A. C. Ferrari and J. Robertson, Phys. Rev. B 64, 075414 (2001).

${ }^{35}$ A. C. Ferrari and J. Robertson, Phys. Rev. B 61, 14095 (2000).

${ }^{36}$ H. Siegle, L. Eckey, A. Hoffmann, C. Thomsen, B. Meyer, D. Schikora, M. Hankeln, and K. Lischka, Solid State Commun. 96, 943 (1995).

${ }^{37}$ M. Cardona, in Light Scattering in Solids II, Vol. 50 of Topics in Applied Physics, edited by M. Cardona and G. Güntherodt (Springer, Berlin, 1982), p. 19.

${ }^{38}$ M. I. Eremets, M. Gauthier, A. Polian, J. C. Chervin, J. M. Besson, G. A. Dubitskii, and Y. Y. Semenova, Phys. Rev. B 52, 8854 (1995).

${ }^{39}$ L. Bergmann and R. J. Nemanich, Annu. Rev. Mater. Sci. 26, 551 (1996).

${ }^{40}$ W. D. Johnston, Jr. and I. P. Kaminov, Phys. Rev. 188, 1209 (1969).

${ }^{41}$ C. Flytzanis, Phys. Rev. B 6, 1264 (1972).

${ }^{42}$ M. V. Klein, in Light Scattering in Solids I, Vol. 8 of Topics in Applied Physics, edited by M. Cardona, 2nd ed. (Springer, Berlin, 1983), p. 147.

${ }^{43}$ V. I. Gavrilenko and R. Q. Wu, Phys. Rev. B 61, 2632 (2000).

${ }^{44}$ J. Chen, L. Jönsson, J. W. Wilkins, and Z. H. Levine, Phys. Rev. B 56, 1787 (1997).

${ }^{45}$ A. Onodera, M. Nakatani, M. Kobayashi, Y. Nisida, and O. Mishima, Phys. Rev. B 48, 2777 (1993).

${ }^{46}$ J. M. Calleja, J. Kuhl, and M. Cardona, Phys. Rev. B 17, 876 (1978).

${ }^{47}$ C. Trallero-Giner, A. Cantarero, M. Cardona, and M. Mora, Phys. Rev. B 45, 6601 (1992).

${ }^{48}$ I. Loa, S. Gronemeyer, C. Thomsen, O. Ambacher, D. Schikora, and D. J. As, J. Raman Spectrosc. 29, 291 (1998).

${ }^{49}$ R. A. Roberts and W. C. Walker, Phys. Rev. 161, 730 (1967).

${ }^{50}$ O. Stenzel, J. Hahn, M. Roder, A. Enrlich, S. Prause, and F. Richter, Phys. Status Solidi A 158, 281 (1996).
${ }^{51}$ C. D. Clark, P. J. Dean, and P. V. Harris, Proc. R. Soc. London, Ser. A 277, 312 (1964).

${ }^{52}$ N. Miyata, K. Moriki, O. Mishima, M. Fujisawa, and T. Hattori, Phys. Rev. B 40, 12028 (1998).

${ }^{53}$ D. M. Hoffman, G. L. Doll, and P. C. Eklund, Phys. Rev. B 30, 6051 (1984).

${ }^{54}$ A. Zunger, A. Katzir, and A. Halperin, Phys. Rev. B 13, 5560 (1976).

${ }^{55}$ J. M. Ralston, R. L. Wadsack, and R. K. Chang, Phys. Rev. Lett. 25, 814 (1970).

${ }^{56}$ P. Rodríguz-Hernández, M. González-Diaz, and A. Muñoz, Phys. Rev. B 51, 14705 (1995).

${ }^{57}$ A. Compaan and H. J. Trodahl, Phys. Rev. B 29, 793 (1984).

${ }^{58}$ M. Djouadi, S. Ilias, D. Bouchier, J. Pascallon, G. Sené, and V. Stambouli, Diamond Relat. Mater. 7, 1657 (1998).

${ }^{59}$ K. Karch and F. Bechstedt, Phys. Rev. B 56, 7404 (1997).

${ }^{60}$ J. Maultzsch, S. Reich, C. Thomsen, H. Requardt, and P. Ordejón, Phys. Rev. Lett. 92, 075501 (2004).

${ }^{61}$ S. Piscanec, M. Lazzeri, F. Mauri, A. C. Ferrari, and J. Robertson, Phys. Rev. Lett. 93, 185503 (2004).

${ }^{62}$ R. J. Nemanich and S. A. Solin, Solid State Commun. 23, 417 (1977).

${ }^{63}$ S. A. Solin and A. K. Ramdas, Phys. Rev. B 1, 1687 (1970).

${ }^{64}$ R. J. Nemanich and S. A. Solin, Phys. Rev. B 20, 392 (1979).

${ }^{65}$ J. Kulda, H. Kainzmaier, D. Strauch, B. Dorner, M. Lorenzen, and M. Krisch, Phys. Rev. B 66, 241202(R) (2002).

${ }^{66}$ N. Ohba, K. Miwa, N. Nagasako, and A. Fukumoto, Phys. Rev. B 63, 115207 (2001).

${ }^{67}$ E. B. Wilson, J. C. Decius, and P. C. Cross, Molecular Vibrations (Dover, New York, 1980).

${ }^{68}$ T. Inui, Y. Tanabe, and Y. Onodera, Group Theory and its Application in Physics (Springer Verlag, Berlin, 1996).

${ }^{69}$ R. Trommer and M. Cardona, Phys. Rev. B 17, 1865 (1973).

${ }^{70}$ B. A. Weinstein and M. Cardona, Phys. Rev. B 7, 2545 (1973).

${ }^{71}$ R. Zedlitz, M. Heintze, and M. B. Schubert, J. Non-Cryst. Solids 198-200, 403 (1996).

${ }^{72}$ X. Zhang, Chem. Phys. Lett. 373, 506 (2003). 